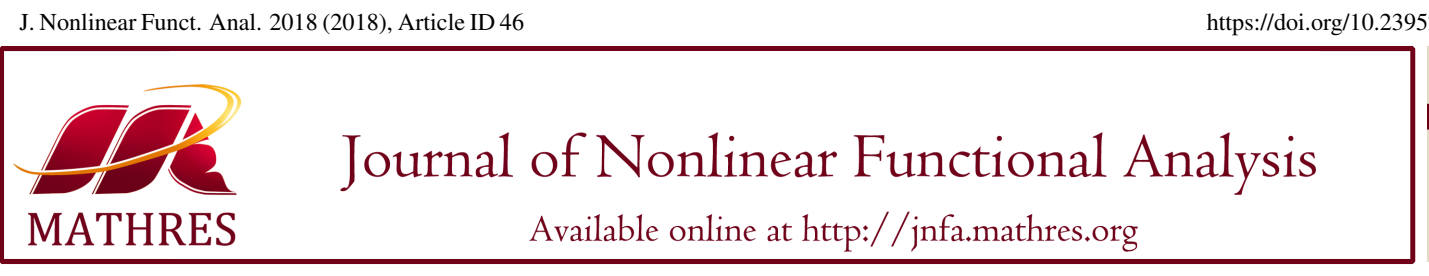

https://doi.org/10.23952/jnfa.2018.46

\title{
A NEW ITERATIVE ALGORITHM FOR SOLVING A SYSTEM OF GENERALIZED EQUILIBRIUM PROBLEMS
}

\author{
A. ABKAR* ${ }^{*}$ M. SHEKARBAIGI, N. AGHAMOHAMMADI
}

Department of Pure Mathematics, Faculty of Science, Imam Khomeini International University, Qazvin 34149, Iran

\begin{abstract}
In this paper, under the framework of real Hilbert spaces, we introduce a new iterative algorithm for finding a common element in the solution set of a generalized equilibrium problem and in the fixed-point sets of a family of nonexpansive mappings. We obtain strong convergence theorems of the common solution problem. An example is provided to support the convergence analysis.
\end{abstract}

Keywords. Iterative algorithm; Generalized equilibrium problem; Variational inequality; Nonexpansive mapping; Strongly monotone operator.

2010 Mathematics Subject Classification. 47H09, 47H10, 47J25.

\section{INTRODUCTION}

Let $\mathscr{H}$ be a real Hilbert space equipped with inner product $\langle\cdot, \cdot\rangle$ and induced norm $\|\cdot\|$. Let $\mathscr{C}$ be a nonempty closed convex subset of $\mathscr{H}$, and let $\Upsilon: \mathscr{C} \times \mathscr{C} \rightarrow \mathbb{R}$ be a bifunction.

The equilibrium problem for $\Upsilon: \mathscr{C} \times \mathscr{C} \rightarrow \mathbb{R}$ is to find $x \in \mathscr{C}$ such that

$$
\Upsilon(x, y) \geq 0, \quad \forall y \in \mathscr{C} .
$$

The solution set of (1.1) is denoted by $E P(\Upsilon)$ in this paper. This equilibrium problem, which was first introduced by Ky Fan [1], and further studied by Blum and Oettli [2], has been extensively investigated based on fixed point methods; see $[3,4,5,6,7,8]$ and the references therein.

In 2005, Combettes and Hirstoaga [9] introduced an iterative scheme for finding the best approximation to the initial data when $E P(\Upsilon)$ is nonempty, and proved a strong convergence theorem. The equilibrium problem has emerged as an effective and powerful tool for studying a wide class of problems which arise in economics, ecology, transportation, network, elasticity and optimization; see [10,11, 12] and the references therein.

\footnotetext{
* Corresponding author.
}

E-mail addresses: abkar@sci.ikiu.ac.ir (A. Abkar), m.shekarbaigi@sci.ikiu.ac.ir(M. Shekarbaigi).

Received March 18, 2018; Accepted December 10, 2018. 
Let $S: \mathscr{C} \rightarrow \mathscr{C}$ be a mapping. Throughout this paper, the set of fixed points of mapping $S$ is denoted by

$$
\operatorname{Fix}(S)=\{x \in \mathscr{C}: x=S x\} .
$$

Recall that $S: \mathscr{C} \rightarrow \mathscr{C}$ is said to be nonexpansive if

$$
\|S x-S y\| \leq\|x-y\|, \quad \forall x, y \in \mathscr{C} .
$$

It was proved in [9] that equilibrium problem (1.1) is equivalent to a fixed point problem of nonexpansive mappings. Let $r>0$ be a positive real number. Define a mapping $T_{r}: \mathscr{H} \rightrightarrows \mathscr{C}$ by

$$
T_{r}(x)=\left\{z \in \mathscr{C}: \Upsilon(z, y)+\frac{1}{r}\langle y-z, z-x\rangle \geq 0, \forall y \in \mathscr{C}\right\}
$$

Then $T_{r}$ is single-valued, and $F i x\left(T_{r}\right)=E P(\Upsilon)$.

Let $A: \mathscr{C} \rightarrow \mathscr{H}$ be a mapping. Recall that $A$ is said to be monotone if

$$
\langle A x-A y, x-y\rangle \geq 0, \quad \forall x, y \in \mathscr{C} .
$$

Recall that $A$ is said to be $\alpha$-inverse strongly monotone if there exists a constant $\alpha>0$ such that

$$
\langle A x-A y, x-y\rangle \geq \alpha\|A x-A y\|^{2}, \forall x, y \in \mathscr{C} .
$$

A bounded linear operator $A$ on $\mathscr{H}$ is said to be strongly positive if there is a $\delta>0$ such that

$$
\langle A x, x\rangle \geq \delta\|x\|^{2}, \quad \forall x \in \mathscr{H} .
$$

Let $A: \mathscr{C} \rightarrow \mathscr{C}$ be an inverse strongly monotone mapping and let $\Upsilon: \mathscr{C} \times \mathscr{C} \rightarrow \mathbb{R}$ be a bifunction. In this paper, we consider the following Generalized Equilibrium Problem (GEP):

$$
\text { Find } \tilde{x} \in \mathscr{C} \text { such that } \Upsilon(\tilde{x}, y)+\langle A \tilde{x}, y-\tilde{x}\rangle \geq 0, \forall y \in \mathscr{C} \text {. }
$$

The set of solutions of this problem is denoted by $\operatorname{GEP}(\Upsilon, A)$, i.e.,

$$
G E P(\Upsilon, A)=\{x \in \mathscr{C}: \Upsilon(x, y)+\langle A x, y-x\rangle \geq 0, \forall y \in \mathscr{C}\}
$$

It is desirable to have a look at some special cases of generalized equilibrium problem (1.2):

(i): if $A \equiv 0$, then problem (1.2) reduces to the following Equilibrium Problem:

Find $\tilde{x} \in \mathscr{C}$ such that $\Upsilon(\tilde{x}, y) \geq 0, \forall y \in \mathscr{C}$;

(ii): if $\Upsilon \equiv 0$, then problem (1.2) reduces to the following Classical Variational Inequality:

Find $\tilde{x} \in \mathscr{C}$ such that $\langle A \tilde{x}, y-\tilde{x}\rangle \geq 0, \forall y \in \mathscr{C}$.

As in the case of equilibrium problem, the classical variational inequality problem is equivalent to a fixed point problem. To see this, we should recall the orthogonal projection $P_{\mathscr{C}}: \mathscr{H} \rightarrow \mathscr{C}$ that assigns $x \in \mathscr{H}$ to its unique nearest point in $\mathscr{C}$ provided that this latter set is nonempty, closed and convex. Indeed, we have

$$
P_{\mathscr{C}}(x)=\arg \min \{\|x-y\|: y \in \mathscr{C}\} .
$$

It is well-known that

$$
y=P_{\mathscr{C}}(x) \Longleftrightarrow\langle x-y, y-z\rangle \geq 0, \quad \forall z \in \mathscr{C} .
$$


We assume that $\lambda>0$ and $u \in \mathscr{C}$. Then $u \in V I(\mathscr{C}, A)$ if and only if $\lambda\langle A u, z-u\rangle \geq 0$ for each $z \in \mathscr{C}$. This in turn is equivalent to

$$
\begin{aligned}
\langle-\lambda A u, u-z\rangle \geq 0, \quad \forall z \in \mathscr{C} & \Longleftrightarrow\langle u-\lambda A u-u, u-z\rangle \geq 0, \quad \forall z \in \mathscr{C} \\
& \Longleftrightarrow\langle(I-\lambda A) u-u, u-z\rangle \geq 0, \quad \forall z \in \mathscr{C} \\
& \Longleftrightarrow u=P_{\mathscr{C}}(I-\lambda A) u .
\end{aligned}
$$

This shows that $u \in \mathscr{C}$ is a solution of the variational inequality if and only if $u$ is a fixed point of $P_{\mathscr{C}}(I-\lambda A)$; where $\lambda>0$ is a constant, and $I$ is the identity operator. Recently, many authors have studied problem (1.2) and its two special cases via fixed-point methods; see $[13,14,15,16,17,18]$ and the references therein.

In [19], Shimoji and Takahashi introduced a $W_{n}$-mapping induced by an infinite family of nonexpansive mappings $\left\{S_{i}: \mathscr{C} \rightarrow \mathscr{C}\right\}$ and a real number sequence of $\left\{\gamma_{i}\right\}$ with the restriction $0 \leq \gamma_{i}<1, \forall i \geq 1$ (see next section). In 2016, Zhang and Hao [13], based on the $W_{n}$-mapping, studied the convergence analysis of the following iterative sequence, which is defined as following:

$$
\left\{\begin{array}{l}
x_{1} \in \mathscr{C}, \text { chosen/arbitrarily, } \\
F\left(y_{n}, y\right)+\left\langle A x_{n}, y-y_{n}\right\rangle+\frac{1}{r_{n}}\left\langle y-y_{n}, y_{n}-x_{n}\right\rangle \geq 0, \forall y \in \mathscr{C}, \\
x_{n+1}=\beta_{n} x_{n}+\left(1-\beta_{n}\right) W_{n}\left(\alpha_{n} f\left(W_{n} x_{n}\right)+\left(1-\alpha_{n}\right) y_{n}\right), \forall n \geq 1,
\end{array}\right.
$$

where $F$ is a bifunction, $f$ is a contraction, $\left\{\alpha_{n}\right\}$ and $\left\{\beta_{n}\right\}$ are sequences in the open interval $(0,1)$ and $\left\{r_{n}\right\}$ is a sequence of positive numbers. They proved that the sequence $\left\{x_{n}\right\}$ converges strongly to a point

$$
x \in \Omega:=\bigcap_{i=1}^{\infty} \operatorname{Fix}\left(S_{i}\right) \cap \operatorname{GEP}(F, A) \neq \emptyset,
$$

where $x=P_{\Omega} f(x)$.

Subsequently, Majee and Nahak [14] studied the convergence analysis of the following iterative sequence $\left\{x_{n}\right\}$, which is defined in the following way: $x_{1} \in \mathscr{C}$ arbitrarily, and

$$
\left\{\begin{array}{l}
u_{n}=T_{r_{n}}^{\left(F_{1}, h_{1}\right)}\left(x_{n}+\gamma A^{*}\left(T_{r_{n}}^{\left(F_{2}, h_{2}\right)}-I\right) A x_{n}\right) \\
y_{n}=\delta_{n} x_{n}+\left(1-\delta_{n}\right) S_{N}^{n} S_{N-1}^{n} \cdots S_{1}^{n} u_{n} \\
x_{n+1}=\alpha_{n} \eta f\left(x_{n}\right)+\beta_{n} x_{n}+\left(\left(1-\beta_{n}\right) I-\alpha_{n} \mu D\right) y_{n}, n \geq 1
\end{array}\right.
$$

where $D$ is a strongly positive bounded linear operator on $\mathscr{H}, F_{1}, F_{2}, h_{1}, h_{2}$ are some bifunctions, $\eta, \mu>$ $0,\left\{r_{n}\right\} \subset(0, \infty)$, and $\left\{\alpha_{n}\right\},\left\{\beta_{n}\right\},\left\{\delta_{n}\right\} \subset[0,1]$.

In this paper, inspired by [13] and [14], we introduce a new iterative algorithm and prove a strong convergence theorem for generalized equilibrium problem (1.2) and common fixed-point problem of a family of nonexpasnive mappings. Comparing with the algorithm in [13], we here consider a system of generalized equilibrium problems (instead of one bifunction and one $\alpha$-inverse strongly monotone operator). It also deserves mentioning that the number of nonexpansive mappings in our algorithm is infinite. The results presented in this paper improve and extend some recent results announced in $[13,14,20,21,22]$. 


\section{PRELIMINARIES}

To study generalized equilibrium problem (1.2), we assume that bifunction $\Upsilon: \mathscr{C} \times \mathscr{C} \rightarrow \mathbb{R}$ satisfies the following conditions:

(C1): $\Upsilon(x, x)=0$ for all $x \in \mathscr{C}$,

(C2): $\Upsilon$ is monotone, that is,

$$
\Upsilon(x, y)+\Upsilon(y, x) \leq 0, \forall x, y \in \mathscr{C},
$$

(C3): $\Upsilon$ is upper-hemicontinuous, that is,

$$
\underset{h \rightarrow 0^{+}}{\limsup } \Upsilon(h z+(1-h) x, y) \leq \Upsilon(x, y), \forall x, y, z \in \mathscr{C},
$$

(C4): $\Upsilon(x, 0)$ is convex and lower semicontinuous for each $x \in \mathscr{C}$.

In order to prove our main results, we need to recall some concepts from the theory of Banach spaces. A Banach space $X$ is said to satisfy the Opial's condition [23] if, for each sequence $\left\{x_{n}\right\}_{n=1}^{\infty}$ in $X$ which converges weakly to a point $x \in X$, we have

$$
\liminf _{n \rightarrow \infty}\left\|x_{n}-x\right\|<\liminf _{n \rightarrow \infty}\left\|x_{n}-y\right\|, \quad \forall y \in X, y \neq x .
$$

It is known that the above inequality is equivalent to

$$
\limsup _{n \rightarrow \infty}\left\|x_{n}-x\right\|<\limsup _{n \rightarrow \infty}\left\|x_{n}-y\right\|, \quad \forall y \in X, y \neq x .
$$

It is known that all Hilbert spaces and $l^{p}$ satisfy the Opial's condition, however, $L^{p}$ does not satisfy the Opial's condition, unless $p=2$.

Let $\mathscr{H}$ be a real Hilbert space. Then, for all $x, y \in \mathscr{H}$, the following assertions hold:

(1) $\|x+y\|^{2} \leq\|y\|^{2}+2\langle x, x+y\rangle$,

(2) $\|\alpha x+(1-\alpha) y\|^{2}=\alpha\|x\|^{2}+(1-\alpha)\|y\|^{2}-\alpha(1-\alpha)\|x-y\|^{2}$,

(3) $\|x-y\|^{2}=\|x\|^{2}+\|y\|^{2}-2\langle x, y\rangle$.

Let $\mathscr{C}$ be a nonempty closed and convex subset of a Hilbert space $\mathscr{H}$. Let $T: \mathscr{C} \rightarrow \mathscr{C}$ be a mapping. Recall that $T$ is said to be firmly nonexpansive iff

$$
\|T x-T y\|^{2} \leq\langle T x-T y, x-y\rangle, \quad x, y \in \mathscr{C} .
$$

It is easy to see that every firmly nonexpansive mapping is nonexpansive. As a typical example, the orthogonal projection $P_{\mathscr{C}}$, which satisfies

$$
\left\|P_{\mathscr{C}} x-P_{\mathscr{C}} y\right\|^{2} \leq\left\langle P_{\mathscr{C}} x-P_{\mathscr{C}} y, x-y\right\rangle, \quad \forall x, y \in \mathscr{C},
$$

is firmly nonexpansive.

Lemma 2.1. [2] Let $\mathscr{C}$ be a nonempty closed and convex subset of a Hilbert space $\mathscr{H}$. Let $\Upsilon: \mathscr{C} \times \mathscr{C} \rightarrow \mathbb{R}$ be a bifunction. Assume that $\Upsilon$ satisfies $(\boldsymbol{C 1})-(\boldsymbol{C 4})$. Let $r>0$ and $x \in \mathscr{H}$. Then, there exists $z \in \mathscr{C}$ such that

$$
\Upsilon(z, y)+\frac{1}{r}\langle y-z, z-x\rangle \geq 0, \forall y \in \mathscr{C} .
$$

Further, define a mapping $T_{r}: \mathscr{H} \rightrightarrows \mathscr{C}$ by

$$
T_{r}(x)=\left\{z \in \mathscr{C}: \Upsilon(z, y)+\frac{1}{r}\langle y-z, z-x\rangle \geq 0, \forall y \in \mathscr{C}\right\} .
$$

Then 
(1) $T_{r}$ is single-valued.

(2) $T_{r}$ is firmly nonexpansive, that is,

$$
\left\|T_{r}(x)-T_{r}(y)\right\|^{2} \leq\left\langle T_{r}(x)-T_{r}(y), x-y\right\rangle, \quad \forall x, y \in \mathscr{H}
$$

(3) $\operatorname{Fix}\left(T_{r}\right)=E P(\Upsilon)$,

(4) $E P(\Upsilon)$ is closed and convex.

Lemma 2.2. [17] Let $\mathscr{C}, \mathscr{H}, \Upsilon$ and $T_{r}$ be as in Lemma 2.1. Then the following holds:

$$
\left\|T_{s}(x)-T_{t}(x)\right\|^{2} \leq \frac{s-t}{s}\left\langle T_{s}(x)-T_{t}(x), T_{s}(x)-x\right\rangle,
$$

for all $s, t>0$ and $x \in \mathscr{H}$.

Lemma 2.3. [24] Assume that $\left\{a_{n}\right\}$ is a sequence of nonnegative real numbers such that

$$
a_{n+1} \leq\left(1-\gamma_{n}\right) a_{n}+\gamma_{n} \delta_{n}, \forall n \geq 0,
$$

where $\left\{\gamma_{n}\right\}$ is a sequence in $(0,1)$ and $\left\{\delta_{n}\right\}$ is a sequence in $\mathbb{R}$ such that

(1) $\sum_{n=1}^{\infty} \gamma_{n}=\infty$,

(2) $\limsup _{n \rightarrow \infty} \frac{\delta_{n}}{\gamma_{n}} \leq 0$ or $\sum_{n=1}^{\infty}\left|\delta_{n}\right|<\infty$.

Then $\lim _{n \rightarrow \infty} a_{n}=0$.

Definition 2.4. [19] Let $\mathscr{C}$ be a nonempty closed convex subset of a Hilbert space $\mathscr{H}$. Let $\left\{S_{i}: \mathscr{C} \rightarrow \mathscr{C}\right\}$ be an infinite family of nonexpansive mappings and let $\left\{\gamma_{i}\right\}$ be a nonnegative real sequence with $0 \leq \gamma_{i}<$ $1, \forall i \geq 1$. For $n \geq 1$, define a mapping $W_{n}: \mathscr{C} \rightarrow \mathscr{C}$ as follows

$$
\left\{\begin{array}{l}
U_{n, n+1}=I \\
U_{n, n}=\gamma_{n} S_{n} U_{n, n+1}+\left(1-\gamma_{n}\right) I \\
U_{n, n-1}=\gamma_{n-1} S_{n-1} U_{n, n}+\left(1-\gamma_{n-1}\right) I \\
\vdots \\
U_{n, k}=\gamma_{k} S_{k} U_{n, k+1}+\left(1-\gamma_{k}\right) I \\
U_{n, k-1}=\gamma_{k-1} S_{k-1} U_{n, k}+\left(1-\gamma_{k-1}\right) I \\
\vdots \\
U_{n, 2}=\gamma_{2} S_{2} U_{n, 3}+\left(1-\gamma_{2}\right) I \\
W_{n}=U_{n, 1}=\gamma_{1} S_{1} U_{n, 2}+\left(1-\gamma_{1}\right) I .
\end{array}\right.
$$

Lemma 2.5. [19] Let $\mathscr{C}$ be a nonempty closed convex subset of a Hilbert space $\mathscr{H}$. Let $\left\{S_{i}: \mathscr{C} \rightarrow \mathscr{C}\right\}$ be an infinite family of nonexpansive mappings with $\cap_{i=1}^{\infty} F i x\left(S_{i}\right) \neq \emptyset$ and let $\left\{\gamma_{i}\right\}$ be a real sequence such that $0 \leq \gamma_{i} \leq l<1, \forall i \geq 1$. Then

(1) $W_{n}$, defined in the previous section, is nonexpansive and Fix $\left(W_{n}\right)=\cap_{i=1}^{\infty} \operatorname{Fix}\left(S_{i}\right)$, for each $n \geq 1$;

(2) for each $x \in \mathscr{C}$ and for each positive integer $k$, the limit $\lim _{n \rightarrow \infty} U_{n, k}$ exists;

(3) the mapping $W: \mathscr{C} \rightarrow \mathscr{C}$ defined by

$$
W x:=\lim _{n \rightarrow \infty} W_{n} x=\lim _{n \rightarrow \infty} U_{n, 1} x, x \in \mathscr{C},
$$

is nonexpansive and satisfies Fix $(W)=\cap_{i=1}^{\infty} \operatorname{Fix}\left(S_{i}\right)$. 
The mapping $W$ defined above is called the $W$-mapping generated by $S_{1}, S_{2}, \cdots$ and $\gamma_{1}, \gamma_{2}, \cdots$.

Lemma 2.6. [20] Let $\mathscr{C}$ be a nonempty closed convex subset of $\mathscr{H},\left\{S_{i}: \mathscr{C} \rightarrow \mathscr{C}\right\}$ be an infinite family of nonexpansive mappings with $\cap_{i=1}^{\infty} F i x\left(S_{i}\right) \neq \emptyset$ and $\left\{\gamma_{i}\right\}$ be a real sequence such that $0 \leq \gamma_{i} \leq l<1, \forall i \geq 1$. If $K$ is any bounded subset of $\mathscr{C}$, then

$$
\lim _{n \rightarrow \infty} \sup _{x \in K}\left\|W x-W_{n} x\right\|=0 .
$$

Throughout this paper, we always assume that $0 \leq \gamma_{i} \leq l<1$, where $l$ is some real number, $i \geq 1$.

Lemma 2.7. [25] Let $\left\{x_{n}\right\}$ and $\left\{y_{n}\right\}$ be bounded sequences in $\mathscr{H}$ and let $\left\{\beta_{n}\right\}$ be a sequence in $(0,1)$ with $0<\liminf _{n \rightarrow \infty} \beta_{n} \leq \limsup _{n \rightarrow \infty} \beta_{n}<1$. Suppose that $x_{n+1}=\left(1-\beta_{n}\right) y_{n}+\beta_{n} x_{n}$ for all $n \geq 0$ and

$$
\limsup _{n \rightarrow \infty}\left(\left\|y_{n+1}-y_{n}\right\|-\left\|x_{n+1}-x_{n}\right\|\right) \leq 0 \text {. }
$$

Then $\lim _{n \rightarrow \infty}\left\|y_{n}-x_{n}\right\|=0$.

\section{Main Results}

Theorem 3.1. Let $\mathscr{C}$ be a nonempty, closed convex subset of a Hilbert space $\mathscr{H}$ and let $\Upsilon_{1}, \Upsilon_{2}, \cdots, \Upsilon_{k}$ be bifunctions from $\mathscr{C} \times \mathscr{C}$ to $\mathbb{R}$ which satisfy $(\boldsymbol{C 1})-(\boldsymbol{C 4})$. Let $A_{1}, A_{2}, \cdots, A_{k}$ be $\mu_{j}$-inverse strongly monotone mappings from $\mathscr{C}$ to $\mathscr{H}$ and let $\left\{S_{i}: \mathscr{C} \rightarrow \mathscr{C}\right\}$ be an infinite family of nonexpansive mappings. Let $f: \mathscr{C} \rightarrow \mathscr{C}$ be a contractive mapping with the constant $k \in(0,1)$. Assume that

$$
\Omega:=\left(\bigcap_{i=1}^{\infty} \operatorname{Fix}\left(S_{i}\right)\right) \cap\left(\bigcap_{j=1}^{k} \operatorname{GEP}\left(\Upsilon_{j}, A_{j}\right)\right) \neq \emptyset
$$

Let $\left\{x_{n}\right\}_{n=1}^{\infty}$ be a sequence generated in the following manner:

$$
\left\{\begin{array}{l}
x_{1} \in \mathscr{C}, y \in \mathscr{C}, \\
\Upsilon_{1}\left(u_{n, 1}, y\right)+\left\langle A_{1} x_{n}, y-u_{n, 1}\right\rangle+\frac{1}{r_{n}}\left\langle y-u_{n, 1}, u_{n, 1}-x_{n}\right\rangle \geq 0 \\
\Upsilon_{2}\left(u_{n, 2}, y\right)+\left\langle A_{2} x_{n}, y-u_{n, 2}\right\rangle+\frac{1}{r_{n}}\left\langle y-u_{n, 2}, u_{n, 2}-x_{n}\right\rangle \geq 0 \\
\vdots \\
\Upsilon_{k}\left(u_{n, k}, y\right)+\left\langle A_{k} x_{n}, y-u_{n, k}\right\rangle+\frac{1}{r_{n}}\left\langle y-u_{n, k}, u_{n, k}-x_{n}\right\rangle \geq 0 \\
\omega_{n}=\frac{1}{k} \sum_{j=1}^{k} u_{n, j}, \\
x_{n+1}=\beta_{n} x_{n}+\left(1-\beta_{n}\right) W_{n}\left(\alpha_{n} f\left(W_{n} x_{n}\right)+\left(1-\alpha_{n}\right) \omega_{n}\right),
\end{array}\right.
$$

where $\left\{W_{n}\right\}$ is the sequence defined by (2.1), $\left\{\alpha_{n}\right\}$ and $\left\{\beta_{n}\right\}$ are sequences in $(0,1)$ and $\left\{r_{n}\right\}$ is a sequence of positive numbers. Assume that the above control sequences satisfy the following conditions: $0<a \leq \beta_{n} \leq b<1,0<c \leq r_{n} \leq d<2 \mu, \lim _{n \rightarrow \infty} \alpha_{n}=0, \sum_{n=1}^{\infty} \alpha_{n}=\infty$ and $\lim _{n \rightarrow \infty}\left(r_{n}-r_{n+1}\right)=0$. Then $\left\{x_{n}\right\}$ convergence strongly to a point $x \in \Omega$, where $x=P_{\Omega} f(x)$. 
Proof. We first show that $\left\{x_{n}\right\}$ and $\left\{\omega_{n}\right\}$ are bounded. Let $x^{*} \in \Omega$. We observe that $I-r_{n} A_{j}, j=$ $1,2, \cdots, k$, is a nonexpansive mapping. Indeed, for any $x, y \in \mathscr{C}$, one has

$$
\begin{aligned}
\left\|\left(I-r_{n} A_{j}\right) x-\left(I-r_{n} A_{j}\right) y\right\|^{2} & =\left\|(x-y)-r_{n}\left(A_{j} x-A_{j} y\right)\right\|^{2} \\
& =\|x-y\|^{2}-2 r_{n}\left\langle x-y, A_{j} x-A_{j} y\right\rangle+r_{n}^{2}\left\|A_{j} x-A_{j} y\right\|^{2} \\
& \leq\|x-y\|^{2}-r_{n}\left(2 \mu_{j}-r_{n}\right)\left\|A_{j} x-A_{j} y\right\|^{2} \\
& \leq\|x-y\|^{2} .
\end{aligned}
$$

So

$$
\left\|u_{n, i}-x^{*}\right\| \leq\left\|x_{n}-x^{*}\right\|
$$

which in turn implies that

$$
\left\|\omega_{n}-x^{*}\right\| \leq\left\|x_{n}-x^{*}\right\| .
$$

Putting $z_{n}=\alpha_{n} f\left(W_{n} x_{n}\right)+\left(1-\alpha_{n}\right) \omega_{n}$, we find from (3.3) that

$$
\begin{aligned}
\left\|z_{n}-x^{*}\right\| & \leq \alpha_{n}\left\|f\left(W_{n} x_{n}\right)-x^{*}\right\|+\left(1-\alpha_{n}\right)\left\|\omega_{n}-x^{*}\right\| \\
& \leq\left(1-\alpha_{n}(1-k)\right)\left\|x_{n}-x^{*}\right\|+\alpha_{n}\left\|f\left(x^{*}\right)-x^{*}\right\| .
\end{aligned}
$$

It follows that

$$
\begin{aligned}
\left\|x_{n+1}-x^{*}\right\| & \leq \beta_{n}\left\|x_{n}-x^{*}\right\|+\left(1-\beta_{n}\right)\left\|W_{n} z_{n}-x^{*}\right\| \\
& \leq \beta_{n}\left\|x_{n}-x^{*}\right\|+\left(1-\beta_{n}\right)\left\|z_{n}-x^{*}\right\| \\
& \leq\left(1-\alpha_{n}\left(1-\beta_{n}\right)(1-k)\right)\left\|x_{n}-x^{*}\right\|+\alpha_{n}\left(1-\beta_{n}\right)\left\|f\left(x^{*}\right)-x^{*}\right\| \\
& \vdots \\
& \leq \max \left\{\left\|x_{1}-x^{*}\right\|, \frac{\left\|f\left(x^{*}\right)-x^{*}\right\|}{1-k}\right\} .
\end{aligned}
$$

Therefore, $\left\{x_{n}\right\}$ is bounded, so are $\left\{\omega_{n}\right\}$ and $\left\{z_{n}\right\}$. Without loss of generality, we may assume that there exists a bounded set $K \subset \mathscr{C}$ such that $x_{n}, y_{n}, z_{n} \in K$. Note that $u_{n, j}$ can be written as $u_{n, j}=T_{r_{n}, j}\left(x_{n}-\right.$ $\left.r_{n} A_{j} x_{n}\right)$. For any $j=1,2, \cdots, k$, one has

$$
\begin{gathered}
\left\|u_{n+1, j}-u_{n, j}\right\| \leq\left\|T_{r_{n+1}, j}\left(I-r_{n+1} A_{j}\right) x_{n+1}-T_{r_{n+1}, j}\left(I-r_{n} A_{j}\right) x_{n}\right\| \\
\quad+\left\|T_{r_{n+1}, j}\left(I-r_{n} A_{j}\right) x_{n}-T_{r_{n}, j}\left(I-r_{n} A_{j}\right) x_{n}\right\| \\
\leq\left\|\left(I-r_{n+1} A_{j}\right) x_{n+1}-\left(I-r_{n} A_{j}\right) x_{n}\right\| \\
\quad+\left\|T_{r_{n+1}, j}\left(I-r_{n} A_{j}\right) x_{n}-T_{r_{n}, j}\left(I-r_{n} A_{j}\right) x_{n}\right\| \\
\leq\left\|x_{n+1}-x_{n}\right\|+\left|r_{n+1}-r_{n}\right|\left\|A_{j} x_{n}\right\| \\
\quad+\frac{r_{n+1}-r_{n}}{r_{n+1}}\left\|T_{r_{n+1}, j}\left(I-r_{n} A_{j}\right) x_{n}-T_{r_{n}, j}\left(I-r_{n} A_{j}\right) x_{n}\right\| .
\end{gathered}
$$

Then

$$
\left\|u_{n+1, j}-u_{n, j}\right\| \leq\left\|x_{n+1}-x_{n}\right\|+2 M_{j}\left|r_{n+1}-r_{n}\right|,
$$

where

$$
M_{j}=\max \left\{\sup \left\{\frac{\left\|T_{r_{n+1}, j}\left(I-r_{n} A_{j}\right) x_{n}-T_{r_{n}, j}\left(I-r_{n} A_{j}\right) x_{n}\right\|}{r_{n+1}}\right\}, \sup \left\{\left\|A_{j} x_{n}\right\|\right\}\right\} .
$$


It follows that

$$
\begin{gathered}
\left\|z_{n+1}-z_{n}\right\| \leq \alpha_{n+1}\left\|f\left(W_{n+1} x_{n+1}\right)-f\left(W_{n} x_{n}\right)\right\|+\left|\alpha_{n+1}-\alpha_{n}\right|\left(\left\|f\left(W_{n} x_{n}\right)\right\|+\left\|\omega_{n}\right\|\right) \\
\quad+\left(1-\alpha_{n+1}\right)\left\|\omega_{n+1}-\omega_{n}\right\| \\
\leq \alpha_{n+1} k\left\|W_{n+1} x_{n+1}-W_{n} x_{n}\right\|+\left|\alpha_{n+1}-\alpha_{n}\right|\left(\left\|f\left(W_{n} x_{n}\right)\right\|+\left\|\omega_{n}\right\|\right) \\
\quad+\left\|\omega_{n+1}-\omega_{n}\right\| .
\end{gathered}
$$

Setting $M=\frac{1}{k} \sum_{j=1}^{k} 2 M_{j} \leq \infty$, we have

$$
\left\|\omega_{n+1}-\omega_{n}\right\| \leq \frac{1}{k} \sum_{j=1}^{k}\left\|u_{n+1, j}-u_{n, j}\right\| \leq\left\|x_{n+1}-x_{n}\right\|+M\left|r_{n+1}-r_{n}\right|,
$$

which shows that

$$
\begin{gathered}
\left\|z_{n+1}-z_{n}\right\| \leq \alpha_{n+1} k\left\|W_{n} x_{n}-W_{n} x_{n}\right\|+\left|\alpha_{n+1}-\alpha_{n}\right|\left(\left\|f\left(W_{n} x_{n}\right)\right\|+\left\|\omega_{n}\right\|\right) \\
+\left[\left\|x_{n+1}-x_{n}\right\|+M\left|r_{n+1}-r_{n}\right|\right]
\end{gathered}
$$

Note that

$$
\begin{aligned}
\left\|W_{n+1} z_{n+1}-W_{n} z_{n}\right\| & =\left\|W_{n+1} z_{n+1}-W z_{n+1}+W z_{n+1}-W z_{n}+W z_{n}-W_{n} z_{n}\right\| \\
& \leq\left\|W_{n+1} z_{n+1}-W z_{n+1}\right\|+\left\|W z_{n+1}-W z_{n}\right\|+\left\|W z_{n}-W_{n} z_{n}\right\| \\
& \leq \sup _{x \in K}\left\{\left\|W_{n+1} x-W x\right\|+\left\|W x-W_{n} x\right\|\right\}+\left\|z_{n+1}-z_{n}\right\| .
\end{aligned}
$$

Combing (3.8) with (3.9) yields that

$$
\begin{aligned}
\left\|W_{n+1} z_{n+1}-W_{n} z_{n}\right\|-\left\|x_{n+1}-x_{n}\right\| \leq \sup _{x \in K}\{ & \left.\left\|W_{n+1} x-W x\right\|+\left\|W x-W_{n} x\right\|\right\} \\
& +\alpha_{n+1} k\left\|W_{n+1} x_{n+1}-W_{n} x_{n}\right\| \\
& +\left|\alpha_{n+1}-\alpha_{n}\right|\left(\left\|f\left(W_{n} x_{n}\right)\right\|+\left\|\omega_{n}\right\|\right) \\
+ & {\left[\left\|x_{n+1}-x_{n}\right\|+M\left|r_{n+1}-r_{n}\right|\right] . }
\end{aligned}
$$

By using Lemma 2.6, we find that

$$
\limsup _{n \rightarrow \infty}\left\{\left\|W_{n+1} z_{n+1}-W_{n} z_{n}\right\|-\left\|x_{n+1}-x_{n}\right\|\right\} \leq 0 .
$$

It follows from Lemma 2.7 that

$$
\lim _{n \rightarrow \infty}\left\|W_{n} z_{n}-x_{n}\right\|=0
$$

Consequently,

$$
\lim _{n \rightarrow \infty}\left\|x_{n+1}-x_{n}\right\|=\lim _{n \rightarrow \infty}\left(1-\beta_{n}\right)\left\|W_{n} z_{n}-x_{n}\right\|=0 .
$$

Moreover, for any $j \in\{1,2, \cdots, k\}$, one has

$$
\begin{aligned}
\left\|u_{n, j}-x^{*}\right\|^{2} & \leq\left\|\left(x_{n}-x^{*}\right)-r_{n}\left(A_{j} x_{n}-A_{j} x^{*}\right)\right\|^{2} \\
& =\left\|x_{n}-x^{*}\right\|^{2}-2 r_{n}\left\langle x_{n}-x^{*}, A_{j} x_{n}-A_{j} x^{*}\right\rangle+r_{n}^{2}\left\|A_{j} x_{n}-A_{j} x^{*}\right\|^{2} \\
& \leq\left\|x_{n}-x^{*}\right\|^{2}-r_{n}\left(2 \mu_{j}-r_{n}\right)\left\|A_{j} x_{n}-A_{j} x^{*}\right\|^{2} .
\end{aligned}
$$


It follows that

$$
\begin{aligned}
\left\|\omega_{n}-x^{*}\right\|^{2} & =\left\|\sum_{j=1}^{k} \frac{1}{k}\left(u_{n, j}-x^{*}\right)\right\|^{2} \\
& \leq \frac{1}{k} \sum_{j=1}^{k}\left\|u_{n, j}-x^{*}\right\|^{2} \\
& \leq\left\|x_{n}-x^{*}\right\|^{2}-\frac{1}{k} \sum_{j=1}^{k} r_{n}\left(2 \mu_{j}-r_{n}\right)\left\|A_{j} x_{n}-A_{j} x^{*}\right\|^{2} .
\end{aligned}
$$

By (3.13), we have

$$
\begin{aligned}
\left\|x_{n+1}-x^{*}\right\|^{2}= & \left\|\beta_{n} x_{n}+\left(1-\beta_{n}\right) W_{n} z_{n}-x^{*}\right\|^{2} \\
\leq & \beta_{n}\left\|x_{n}-x^{*}\right\|^{2}+\left(1-\beta_{n}\right)\left\|z_{n}-x^{*}\right\|^{2} \\
\leq & \beta_{n}\left\|x_{n}-x^{*}\right\|^{2}+\left(1-\beta_{n}\right)\left[\left(\alpha_{n}\left\|f\left(W_{n} x_{n}\right)-x^{*}\right\|^{2}+\left(1-\alpha_{n}\right)\left\|\omega_{n}-x^{*}\right\|^{2}\right)\right] \\
\leq & \beta_{n}\left\|x_{n}-x^{*}\right\|^{2}+\left(1-\beta_{n}\right) \alpha_{n}\left\|f\left(W_{n} x_{n}\right)-x^{*}\right\|^{2} \\
& \quad+\left(1-\beta_{n}\right)\left(1-\alpha_{n}\right)\left[\left\|x_{n}-x^{*}\right\|^{2}-\frac{1}{k} \sum_{j=1}^{k} r_{n}\left(2 \mu_{j}-r_{n}\right)\left\|A_{j} x_{n}-A_{j} x^{*}\right\|^{2}\right] \\
& \quad\left\|x_{n}-x^{*}\right\|^{2}+\alpha_{n}\left\|f\left(W_{n} x_{n}\right)-x^{*}\right\|^{2}-\left(1-\alpha_{n}\right)\left(1-\beta_{n}\right) \frac{1}{k} \sum_{j=1}^{k} r_{n}\left(2 \mu_{j}-r_{n}\right)\left\|A_{j} x_{n}-A_{j} x^{*}\right\|^{2},
\end{aligned}
$$

which implies that

$$
\begin{aligned}
& \left(1-\alpha_{n}\right)\left(1-\beta_{n}\right) \frac{1}{k} \sum_{j=1}^{k} r_{n}\left(2 \mu_{j}-r_{n}\right)\left\|A_{j} x_{n}-A_{j} x^{*}\right\|^{2} \\
& \leq\left\|x_{n}-x^{*}\right\|^{2}-\left\|x_{n+1}-x^{*}\right\|^{2}+\alpha_{n}\left\|f\left(W_{n} x_{n}\right)-x^{*}\right\|^{2} \\
& \leq\left(\left\|x_{n}-x^{*}\right\|+\left\|x_{n+1}-x^{*}\right\|\right)\left\|x_{n}-x_{n+1}\right\|+\alpha_{n}\left\|f\left(W_{n} x_{n}\right)-x^{*}\right\|^{2} .
\end{aligned}
$$

Since $\alpha_{n} \rightarrow 0$ and $\left\|x_{n+1}-x_{n}\right\| \rightarrow 0$, we arrive at

$$
\lim _{n \rightarrow \infty}\left\|A_{j} x_{n}-A_{j} x^{*}\right\|=0, \quad \forall j=1,2, \cdots, k .
$$

Note that

$$
\begin{aligned}
\left\|u_{n, j}-x^{*}\right\|^{2} \leq & \left\langle\left(I-r_{n} A_{j}\right) x_{n}-\left(I-r_{n} A_{j}\right) x^{*}, u_{n, j}-x^{*}\right\rangle \\
= & \frac{1}{2}\left(\left\|\left(I-r_{n} A_{j}\right) x_{n}-\left(I-r_{n} A_{j}\right) x^{*}\right\|^{2}+\left\|u_{n, j}-x^{*}\right\|^{2}\right. \\
& \left.\quad-\left\|\left(I-r_{n} A_{j}\right) x_{n}-\left(I-r_{n} A_{j}\right) x^{*}-\left(u_{n, j}-x^{*}\right)\right\|^{2}\right) \\
\leq & \frac{1}{2}\left(\left\|x_{n}-x^{*}\right\|^{2}+\left\|u_{n, j}-x^{*}\right\|^{2}-\left\|x_{n}-u_{n, j}-r_{n}\left(A_{j} x_{n}-A_{j}\right) x^{*}\right\|^{2}\right) \\
= & \frac{1}{2}\left(\left\|x_{n}-x^{*}\right\|^{2}+\left\|u_{n, j}-x^{*}\right\|^{2}-\left\|x_{n}-u_{n, j}\right\|^{2}\right. \\
& \left.\quad+2 r_{n}\left\langle x_{n}-u_{n, j}, A_{j} x_{n}-A_{j} x^{*}\right\rangle-r_{n}^{2}\left\|A_{j} x_{n}-A_{j} x^{*}\right\|^{2}\right) .
\end{aligned}
$$


This implies that

$$
\left\|u_{n, j}-x^{*}\right\|^{2} \leq\left\|x_{n}-x^{*}\right\|^{2}-\left\|x_{n}-u_{n, j}\right\|^{2}+2 r_{n}\left\|x_{n}-u_{n, j}\right\|\left\|A_{j} x_{n}-A_{j} x^{*}\right\| .
$$

It follows that

$$
\begin{aligned}
\left\|\omega_{n}-x^{*}\right\|^{2} & =\left\|\sum_{j=1}^{k} \frac{1}{k}\left(u_{n, j}-x^{*}\right)\right\|^{2} \\
& \leq \frac{1}{k} \sum_{j=1}^{k}\left\|u_{n, j}-x^{*}\right\|^{2} \\
& \leq\left\|x_{n}-x^{*}\right\|^{2}-\frac{1}{k} \sum_{j=1}^{k}\left\|u_{n, j}-x_{n}\right\|^{2}+\frac{1}{k} \sum_{j=1}^{k} 2 r_{n}\left\|x_{n}-u_{n, j}\right\|\left\|A_{j} x_{n}-A_{j} x^{*}\right\| .
\end{aligned}
$$

Observe that

$$
\begin{gathered}
\left\|x_{n+1}-x^{*}\right\|^{2} \leq \beta_{n}\left\|x_{n}-x^{*}\right\|^{2}+\left(1-\beta_{n}\right)\left(\alpha_{n}\left\|f\left(W_{n} x_{n}\right)-x^{*}\right\|^{2}+\left(1-\alpha_{n}\right)\left\|\omega_{n}-x^{*}\right\|^{2}\right) \\
\leq \beta_{n}\left\|x_{n}-x^{*}\right\|^{2}+\alpha_{n}\left(1-\beta_{n}\right)\left\|f\left(W_{n} x_{n}\right)-x^{*}\right\|^{2} \\
+\left(1-\alpha_{n}\right)\left(1-\beta_{n}\right)\left(\left\|x_{n}-x^{*}\right\|^{2}-\frac{1}{k} \sum_{j=1}^{k}\left\|u_{n, j}-x_{n}\right\|^{2}\right. \\
\left.+\frac{1}{k} \sum_{j=1}^{k} 2 r_{n}\left\|x_{n}-u_{n, j}\right\|\left\|A_{j} x_{n}-A_{j} x^{*}\right\|\right) \\
\leq\left\|x_{n}-x^{*}\right\|^{2}+\alpha_{n}\left\|f\left(W_{n} x_{n}\right)-x^{*}\right\|^{2}-\left(1-\alpha_{n}\right)\left(1-\beta_{n}\right) \frac{1}{k} \sum_{j=1}^{k}\left\|u_{n, j}-x_{n}\right\|^{2} \\
+\left(1-\alpha_{n}\right)\left(1-\beta_{n}\right) \frac{1}{k} \sum_{j=1}^{k} 2 r_{n}\left\|x_{n}-u_{n, j}\right\|\left\|A_{j} x_{n}-A_{j} x^{*}\right\| .
\end{gathered}
$$

It follows that

$$
\begin{aligned}
\left(1-\alpha_{n}\right)\left(1-\beta_{n}\right) \frac{1}{k} \sum_{j=1}^{k}\left\|u_{n, j}-x_{n}\right\|^{2} \leq & \alpha_{n}\left\|f\left(W_{n} x_{n}\right)-x^{*}\right\|^{2}+\left\|x_{n}-x^{*}\right\|^{2}-\left\|x_{n+1}-x^{*}\right\|^{2} \\
& +\left(1-\alpha_{n}\right)\left(1-\beta_{n}\right) \frac{1}{k} \sum_{j=1}^{k} 2 r_{n}\left\|x_{n}-u_{n, j}\right\|\left\|A_{j} x_{n}-A_{j} x^{*}\right\| .
\end{aligned}
$$

Since $\alpha_{n} \rightarrow 0$ and $\left\|x_{n+1}-x_{n}\right\| \rightarrow 0$, we have

$$
\lim _{n \rightarrow \infty}\left\|u_{n, j}-x_{n}\right\|=0
$$

It is easy to see that

$$
\lim _{n \rightarrow \infty}\left\|\omega_{n}-x_{n}\right\|=0
$$

Since $z_{n}=\alpha_{n} f\left(W_{n} x_{n}\right)+\left(1-\alpha_{n}\right) \omega_{n}$, we find that

$$
\lim _{n \rightarrow \infty}\left\|z_{n}-\omega_{n}\right\|=0
$$

Notice that

$$
\left\|x_{n+1}-x_{n}\right\|=\left(1-\beta_{n}\right)\left\|W_{n} z_{n}-x_{n}\right\| .
$$


This together with (3.12) gives that

$$
\lim _{n \rightarrow \infty}\left\|W_{n} z_{n}-x_{n}\right\|=0
$$

Observe that

$$
\left\|W_{n} z_{n}-z_{n}\right\| \leq\left\|z_{n}-\omega_{n}\right\|+\left\|\omega_{n}-x_{n}\right\|+\left\|x_{n}-W_{n} z_{n}\right\| .
$$

From (3.18), (3.19), (3.20), we obtain that

$$
\lim _{n \rightarrow \infty}\left\|W_{n} z_{n}-z_{n}\right\|=0 .
$$

Since the mapping $P_{\Omega} f$ is contractive, we denote its unique fixed point by $x$. Next, we prove that

$$
\limsup _{n \rightarrow \infty}\left\langle f(x)-x, z_{n}-x\right\rangle \leq 0 .
$$

To see this, we choose a subsequence $\left\{z_{n_{m}}\right\}$ of $\left\{z_{n}\right\}$ such that

$$
\limsup _{n \rightarrow \infty}\left\langle f(x)-x, z_{n}-x\right\rangle=\lim _{m \rightarrow \infty}\left\langle f(x)-x, z_{n_{m}}-x\right\rangle .
$$

Since $\left\{z_{n_{m}}\right\}$ is bounded, there exists a subsequence $\left\{z_{n_{m_{i}}}\right\}$ of $\left\{z_{n_{m}}\right\}$ which converges weakly to $z$. Without loss of generality, we may assume that $z_{n_{m}} \rightarrow z$. Indeed, we also have $\omega_{n_{m}} \rightarrow f$. First, we show that $z \in \bigcap_{i=1}^{\infty} \operatorname{Fix}\left(S_{i}\right)$. Suppose to the contrary that $W z \neq z$. Note that

$$
\begin{aligned}
\left\|z_{n}-W z_{n}\right\| & \leq\left\|W z_{n}-W_{n} z_{n}\right\|+\left\|W_{n} z_{n}-z_{n}\right\| \\
& \leq \sup _{x \in K}\left\{\left\|W x-W_{n} x\right\|\right\}+\left\|W_{n} z_{n}-z_{n}\right\| .
\end{aligned}
$$

In view of Lemma 2.6, we obtain from (3.21) that $\lim _{n \rightarrow \infty}\left\|z_{n}-W z_{n}\right\|=0$. By using the Opial's condition, we see that

$$
\begin{aligned}
\liminf _{m \rightarrow \infty}\left\|z_{n_{m}}-z\right\| & <\liminf _{m \rightarrow \infty}\left\|z_{n_{m}}-W z\right\| \\
& \leq \liminf _{m \rightarrow \infty}\left\{\left\|z_{n_{m}}-W z_{n_{m}}\right\|+\left\|W z_{n_{m}}-W z\right\|\right\} \\
& \leq \liminf _{m \rightarrow \infty}\left\{\left\|z_{n_{m}}-W z_{n_{m}}\right\|+\left\|z_{n_{m}}-z\right\|\right\}
\end{aligned}
$$

This implies that

$$
\liminf _{m \rightarrow \infty}\left\|z_{n_{m}}-z\right\|<\liminf _{m \rightarrow \infty}\left\|z_{n_{m}}-z\right\|,
$$

which is a contradiction. Hence, we have $z \in \bigcap_{i=1}^{\infty} \operatorname{Fix}\left(S_{i}\right)$.

Next, we show that $f \in \bigcap_{j=1}^{k} G E P\left(\Upsilon_{j}, A_{j}\right)$. Since $\left\{\omega_{n}\right\}$ is bounded, there exists a subsequence $\left\{\omega_{n_{m}}\right\}$ of $\left\{\omega_{n}\right\}$ such that $\omega_{n_{m}} \rightarrow f$. Furthermore, we have

$$
\Upsilon_{j}\left(u_{n, j}, y\right)+\left\langle A_{j} x_{n}, y-u_{n, j}\right\rangle+\frac{1}{r_{n}}\left\langle y-u_{n, j}, u_{n, j}-x_{n}\right\rangle \geq 0, \quad \forall y \in \mathscr{C} .
$$

By using condition $(\mathbf{C 2})$, we see that

$$
\left\langle A_{j} x_{n}, y-u_{n, j}\right\rangle+\frac{1}{r_{n}}\left\langle y-u_{n, j}, u_{n, j}-x_{n}\right\rangle \geq \Upsilon_{j}\left(y, u_{n, j}\right), \quad \forall y \in \mathscr{C} .
$$

Substituting $n$ by $n_{m}$, we get that

$$
\left\langle A_{j} x_{n_{m}}, y-u_{n_{m}, j}\right\rangle+\left\langle y-u_{n_{m}, j}, \frac{u_{n_{m}, j}-x_{n_{m}}}{r_{n_{m}}}\right\rangle \geq \Upsilon_{j}\left(y, u_{n_{m}, j}\right), \quad \forall y \in \mathscr{C} .
$$


For $0<l \leq 1$ and $y \in \mathscr{C}$, let $y_{l}=l y+(1-l) z$. Since $y \in \mathscr{C}$ and $z \in \mathscr{C}$, we have $y_{l} \in \mathscr{C}$. It follows from (3.22) that

$$
\begin{gathered}
\left\langle y_{l}-u_{n_{m}, j}, A_{j} y_{l}\right\rangle \geq\left\langle y_{l}-u_{n_{m}, j}, A_{j} y_{l}\right\rangle-\left\langle A_{j} x_{n_{m}}, y_{l}-u_{n_{m}, j}\right\rangle \\
-\left\langle y_{l}-u_{n_{m}, j}, \frac{u_{n_{m}, j}-x_{n_{m}}}{r_{n_{m}}}\right\rangle+\Upsilon_{j}\left(y_{l}, u_{n_{m}, j}\right) \\
=\left\langle y_{l}-u_{n_{m}, j}, A_{j} y_{l}-A_{j} u_{n_{m}, j}\right\rangle+\left\langle y_{l}-u_{n_{m}, j}, A_{j} u_{n_{m}, j}-A_{j} x_{n_{m}}\right\rangle \\
\quad-\left\langle y_{l}-u_{n_{m}, j}, \frac{u_{n_{m}, j}-x_{n_{m}}}{r_{n_{m}}}\right\rangle+\Upsilon_{j}\left(y_{l}, u_{n_{m}, j}\right) .
\end{gathered}
$$

Monotonicity of $A_{j}$, condition (C4) and (3.17) imply that

$$
\left\langle y_{l}-u_{n_{m}, j}, A_{j} y_{l}-A_{j} u_{n_{m}, j}\right\rangle \geq 0,
$$

and

$$
\left\|A_{j} u_{n_{m}, j}-A_{j} x_{n_{m}}\right\| \rightarrow 0 \text { as } m \rightarrow \infty .
$$

It follows from $(\mathbf{C 4})$ and (3.23) that

$$
\left\langle y_{l}-z, A_{j} y_{l}\right\rangle \geq \Upsilon_{j}\left(y_{l}, z\right) .
$$

Now, (C1) and (C4) together with (3.24) show that

$$
\begin{aligned}
0 & =\Upsilon_{j}\left(y_{l}, y_{l}\right) \leq l \Upsilon_{j}\left(y_{l}, y\right)+(1-l) \Upsilon_{j}\left(y_{l}, z\right) \\
& \leq l \Upsilon_{j}\left(y_{l}, y\right)+(1-l)\left\langle y_{l}-z, A_{j} y_{l}\right\rangle \\
& =l \Upsilon_{j}\left(y_{l}, y\right)+(1-l) l\left\langle y-z, A_{j} y_{l}\right\rangle,
\end{aligned}
$$

which yields $\Upsilon_{j}\left(y_{l}, y\right)+(1-l)\left\langle y-z, A_{j} y_{l}\right\rangle \geq 0$. By letting $l \rightarrow 0$, we have

$$
\Upsilon_{j}(z, y)+\left\langle y-z, A_{j} z\right\rangle \geq 0 .
$$

This shows that $f \in G E P\left(\Upsilon_{j}, A_{j}\right)$ for all $j=1,2, \cdots, k$, or $f \in \bigcap_{j=1}^{k} G E P\left(\Upsilon_{j}, A_{j}\right)$. It follows that

$$
\limsup _{n \rightarrow \infty}\left\langle f(x)-x, z_{n}-x\right\rangle \leq 0 \text {. }
$$

Finally, we show that $x_{n} \rightarrow x$, as $n \rightarrow \infty$. Note that

$$
\begin{aligned}
\left\|z_{n}-x\right\|^{2} & =\alpha_{n}\left\langle f\left(W_{n} x_{n}\right)-x, z_{n}-x\right\rangle+\left(1-\alpha_{n}\right)\left\langle\omega_{n}-x, z_{n}-x\right\rangle \\
& \leq\left(1-\alpha_{n}(1-k)\right)\left\|x_{n}-x\right\|\left\|z_{n}-x\right\|+\alpha_{n}\left\langle f(x)-x, z_{n}-x\right\rangle \\
& \leq \frac{1-\alpha_{n}(1-k)}{2}\left(\left\|x_{n}-x\right\|^{2}+\left\|z_{n}-x\right\|^{2}\right)+\alpha_{n}\left\langle f(x)-x, z_{n}-x\right\rangle .
\end{aligned}
$$

Hence, we have

$$
\left\|z_{n}-x\right\|^{2} \leq\left(1-\alpha_{n}(1-k)\right)\left\|x_{n}-x\right\|^{2}+2 \alpha_{n}\left\langle f(x)-x, z_{n}-x\right\rangle .
$$

This implies that

$$
\begin{aligned}
\left\|x_{n+1}-x\right\|^{2} & =\left\|\beta_{n} x_{n}+\left(1-\beta_{n}\right) W_{n} z_{n}-x\right\|^{2} \\
& \leq \beta_{n}\left\|x_{n}-x\right\|^{2}+\left(1-\beta_{n}\right)\left\|z_{n}-x\right\|^{2} \\
& \leq\left(1-\alpha_{n}\left(1-\beta_{n}\right)(1-k)\right)\left\|x_{n}-x\right\|^{2}+2 \alpha_{n}\left(1-\beta_{n}\right)\left\langle f(x)-x, z_{n}-x\right\rangle .
\end{aligned}
$$

By using Lemma 2.3 and (3.25), we find that $\lim _{n \rightarrow \infty}\left\|x_{n}-x\right\|=0$. This completes proof. 


\section{Applications AND Numerical Examples}

In this section, we give some special cases of 3.1 and numerical results to support the convergence analysis of our algorithm.

For a single nonexpansive mapping, we find from Theorem 3.1 the following result immediately.

Corollary 4.1. Let $\mathscr{C}$ be a nonempty, closed convex subset of a Hilbert space $\mathscr{H}$ and let $\Upsilon_{1}, \Upsilon_{2}, \cdots, \Upsilon_{k}$ be bifunctions from $\mathscr{C} \times \mathscr{C}$ to $\mathbb{R}$ which satisfy $(\boldsymbol{C 1})-(\boldsymbol{C 4})$. Let $A_{1}, A_{2}, \cdots, A_{k}$ be $\mu_{j}$-inverse strongly monotone mappings from $\mathscr{C}$ to $\mathscr{H}$ and let $S: \mathscr{C} \rightarrow \mathscr{C}$ be a nonexpansive mapping. Let $f: \mathscr{C} \rightarrow \mathscr{C}$ be a contractive mapping with the constant $k \in(0,1)$. Assume that

$$
\Omega:=(\operatorname{Fix}(S)) \cap\left(\bigcap_{j=1}^{k} \operatorname{GEP}\left(\Upsilon_{j}, A_{j}\right)\right) \neq \emptyset .
$$

Let $\left\{x_{n}\right\}_{n=1}^{\infty}$ be a sequence generated in the following manner:

$$
\left\{\begin{array}{l}
x_{1} \in \mathscr{C}, y \in \mathscr{C}, \\
\Upsilon_{1}\left(u_{n, 1}, y\right)+\left\langle A_{1} x_{n}, y-u_{n, 1}\right\rangle+\frac{1}{r_{n}}\left\langle y-u_{n, 1}, u_{n, 1}-x_{n}\right\rangle \geq 0 \\
\Upsilon_{2}\left(u_{n, 2}, y\right)+\left\langle A_{2} x_{n}, y-u_{n, 2}\right\rangle+\frac{1}{r_{n}}\left\langle y-u_{n, 2}, u_{n, 2}-x_{n}\right\rangle \geq 0 \\
\vdots \\
\Upsilon_{k}\left(u_{n, k}, y\right)+\left\langle A_{k} x_{n}, y-u_{n, k}\right\rangle+\frac{1}{r_{n}}\left\langle y-u_{n, k}, u_{n, k}-x_{n}\right\rangle \geq 0 \\
\omega_{n}=\frac{1}{k} \sum_{j=1}^{k} u_{n, j}, \\
x_{n+1}=\beta_{n} x_{n}+\left(1-\beta_{n}\right) S\left(\alpha_{n} f\left(S x_{n}\right)+\left(1-\alpha_{n}\right) \omega_{n}\right)
\end{array}\right.
$$

where $\left\{\alpha_{n}\right\}$ and $\left\{\beta_{n}\right\}$ are sequences in $(0,1)$ and $\left\{r_{n}\right\}$ is a sequence of positive numbers. Assume that the above control sequences satisfy the following conditions: $0<a \leq \beta_{n} \leq b<1,0<c \leq r_{n} \leq d<2 \mu$, $\lim _{n \rightarrow \infty} \alpha_{n}=0, \sum_{n=1}^{\infty} \alpha_{n}=\infty$ and $\lim _{n \rightarrow \infty}\left(r_{n}-r_{n+1}\right)=0$. Then $\left\{x_{n}\right\}$ convergence strongly to a point $x \in \Omega$, where $x=P_{\Omega} f(x)$.

Let $S$ be the identity mapping. For a system of generalized equilibrium problems, we find from Theorem 3.1 the following result immediately.

Corollary 4.2. Let $\mathscr{C}$ be a nonempty, closed convex subset of a Hilbert space $\mathscr{H}$ and let $\Upsilon_{1}, \Upsilon_{2}, \cdots, \Upsilon_{k}$ be bifunctions from $\mathscr{C} \times \mathscr{C}$ to $\mathbb{R}$ which satisfy $(\boldsymbol{C 1})-(\boldsymbol{C 4})$. Let $A_{1}, A_{2}, \cdots, A_{k}$ be $\mu_{j}$-inverse strongly monotone mappings from $\mathscr{C}$ to $\mathscr{H}$ and let $f: \mathscr{C} \rightarrow \mathscr{C}$ be a contractive mapping with the constant $k \in$ $(0,1)$. Assume that

$$
\Omega:=\left(\bigcap_{j=1}^{k} \operatorname{GEP}\left(\Upsilon_{j}, A_{j}\right)\right) \neq \emptyset .
$$


Let $\left\{x_{n}\right\}_{n=1}^{\infty}$ be a sequence generated in the following manner:

$$
\left\{\begin{array}{l}
x_{1} \in \mathscr{C}, y \in \mathscr{C} \\
\Upsilon_{1}\left(u_{n, 1}, y\right)+\left\langle A_{1} x_{n}, y-u_{n, 1}\right\rangle+\frac{1}{r_{n}}\left\langle y-u_{n, 1}, u_{n, 1}-x_{n}\right\rangle \geq 0 \\
\Upsilon_{2}\left(u_{n, 2}, y\right)+\left\langle A_{2} x_{n}, y-u_{n, 2}\right\rangle+\frac{1}{r_{n}}\left\langle y-u_{n, 2}, u_{n, 2}-x_{n}\right\rangle \geq 0 \\
\vdots \\
\Upsilon_{k}\left(u_{n, k}, y\right)+\left\langle A_{k} x_{n}, y-u_{n, k}\right\rangle+\frac{1}{r_{n}}\left\langle y-u_{n, k}, u_{n, k}-x_{n}\right\rangle \geq 0 \\
\omega_{n}=\frac{1}{k} \sum_{j=1}^{k} u_{n, j}, \\
x_{n+1}=\beta_{n} x_{n}+\left(1-\beta_{n}\right)\left(\alpha_{n} f\left(x_{n}\right)+\left(1-\alpha_{n}\right) \omega_{n}\right),
\end{array}\right.
$$

where $\left\{\alpha_{n}\right\}$ and $\left\{\beta_{n}\right\}$ are sequences in $(0,1)$ and $\left\{r_{n}\right\}$ is a sequence of positive numbers. Assume that the above control sequences satisfy the following conditions: $0<a \leq \beta_{n} \leq b<1,0<c \leq r_{n} \leq d<2 \mu$, $\lim _{n \rightarrow \infty} \alpha_{n}=0, \sum_{n=1}^{\infty} \alpha_{n}=\infty$ and $\lim _{n \rightarrow \infty}\left(r_{n}-r_{n+1}\right)=0$. Then $\left\{x_{n}\right\}$ convergence strongly to a point $x \in \Omega$, where $x=P_{\Omega} f(x)$.

For a system of equilibrium problems and common fixed point problems of nonexpansive mappings, we find from Theorem 3.1 the following result immediately.

Corollary 4.3. Let $\mathscr{C}$ be a nonempty, closed convex subset of a Hilbert space $\mathscr{H}$ and let $\Upsilon_{1}, \Upsilon_{2}, \cdots, \Upsilon_{k}$ be bifunctions from $\mathscr{C} \times \mathscr{C}$ to $\mathbb{R}$ which satisfy $(\boldsymbol{C 1})-(\boldsymbol{C} 4)$. Let $\left\{S_{i}: \mathscr{C} \rightarrow \mathscr{C}\right\}$ be an infinite family of nonexpansive mappings and let $f: \mathscr{C} \rightarrow \mathscr{C}$ be a contractive mapping with the constant $k \in(0,1)$. Assume that

$$
\Omega:=\left(\bigcap_{i=1}^{\infty} \operatorname{Fix}\left(S_{i}\right)\right) \cap\left(\bigcap_{j=1}^{k} \operatorname{GEP}\left(\Upsilon_{j}, A_{j}\right)\right) \neq \emptyset .
$$

Let $\left\{x_{n}\right\}_{n=1}^{\infty}$ be a sequence generated in the following manner:

$$
\left\{\begin{array}{l}
x_{1} \in \mathscr{C}, y \in \mathscr{C} \\
\Upsilon_{1}\left(u_{n, 1}, y\right)+\frac{1}{r_{n}}\left\langle y-u_{n, 1}, u_{n, 1}-x_{n}\right\rangle \geq 0 \\
\Upsilon_{2}\left(u_{n, 2}, y\right)+\frac{1}{r_{n}}\left\langle y-u_{n, 2}, u_{n, 2}-x_{n}\right\rangle \geq 0 \\
\vdots \\
\Upsilon_{k}\left(u_{n, k}, y\right)+\frac{1}{r_{n}}\left\langle y-u_{n, k}, u_{n, k}-x_{n}\right\rangle \geq 0 \\
\omega_{n}=\frac{1}{k} \sum_{j=1}^{k} u_{n, j} \\
x_{n+1}=\beta_{n} x_{n}+\left(1-\beta_{n}\right) W_{n}\left(\alpha_{n} f\left(W_{n} x_{n}\right)+\left(1-\alpha_{n}\right) \omega_{n}\right)
\end{array}\right.
$$

where $\left\{W_{n}\right\}$ is the sequence defined by (2.1), $\left\{\alpha_{n}\right\}$ and $\left\{\beta_{n}\right\}$ are sequences in $(0,1)$ and $\left\{r_{n}\right\}$ is a sequence of positive numbers. Assume that the above control sequences satisfy the following conditions: $0<a \leq \beta_{n} \leq b<1,0<c \leq r_{n} \leq d<2 \mu, \lim _{n \rightarrow \infty} \alpha_{n}=0, \sum_{n=1}^{\infty} \alpha_{n}=\infty$ and $\lim _{n \rightarrow \infty}\left(r_{n}-r_{n+1}\right)=0$. Then $\left\{x_{n}\right\}$ convergence strongly to a point $x \in \Omega$, where $x=P_{\Omega} f(x)$.

Corollary 4.4. Let $\mathscr{C}$ be a nonempty, closed convex subset of a Hilbert space $\mathscr{H}$ and let $\Upsilon_{1}, \Upsilon_{2}, \cdots, \Upsilon_{k}$ be bifunctions from $\mathscr{C} \times \mathscr{C}$ to $\mathbb{R}$ which satisfy $(\boldsymbol{C 1})-(\boldsymbol{C 4})$. Let $A_{1}, A_{2}, \cdots, A_{k}$ be $\mu_{j}$-inverse strongly 
monotone mappings from $\mathscr{C}$ to $\mathscr{H}$ and let $\left\{S_{i}: \mathscr{C} \rightarrow \mathscr{C}\right\}$ be an infinite family of nonexpansive mappings. Let $f: \mathscr{C} \rightarrow \mathscr{C}$ be a contractive mapping with the constant $k \in(0,1)$. Assume that

$$
\Omega:=\left(\bigcap_{i=1}^{\infty} \operatorname{Fix}\left(S_{i}\right)\right) \cap\left(\bigcap_{j=1}^{k} \operatorname{VI}\left(\mathscr{C}, A_{j}\right)\right) \neq \emptyset .
$$

Let $\left\{x_{n}\right\}_{n=1}^{\infty}$ be a sequence generated in the following manner:

$$
\left\{\begin{array}{l}
x_{1} \in \mathscr{C} \\
u_{n, 1}=P_{\mathscr{C}}\left(x_{n}-r_{n} A_{1} x_{n}\right), \\
u_{n, 2}=P_{\mathscr{C}}\left(x_{n}-r_{n} A_{2} x_{n}\right), \\
\vdots \\
u_{n, k}=P_{\mathscr{C}}\left(x_{n}-r_{n} A_{k} x_{n}\right), \\
\omega_{n}=\frac{1}{k} \sum_{j=1}^{k} u_{n, j}, \\
x_{n+1}=\beta_{n} x_{n}+\left(1-\beta_{n}\right) W_{n}\left(\alpha_{n} f\left(W_{n} x_{n}\right)+\left(1-\alpha_{n}\right) \omega_{n}\right),
\end{array}\right.
$$

where $\left\{W_{n}\right\}$ is the sequence defined by (2.1), $\left\{\alpha_{n}\right\}$ and $\left\{\beta_{n}\right\}$ are sequences in $(0,1)$ and $\left\{r_{n}\right\}$ is a sequence of positive numbers. Assume that the above control sequences satisfy the following conditions: $0<a \leq \beta_{n} \leq b<1,0<c \leq r_{n} \leq d<2 \mu, \lim _{n \rightarrow \infty} \alpha_{n}=0, \sum_{n=1}^{\infty} \alpha_{n}=\infty$ and $\lim _{n \rightarrow \infty}\left(r_{n}-r_{n+1}\right)=0$. Then $\left\{x_{n}\right\}$ convergence strongly to a point $x \in \Omega$, where $x=P_{\Omega} f(x)$.

Proof. Putting $\Upsilon \equiv 0$, we see from Theorem 3.1 that

$$
\left\langle A_{j} x_{n}, y-u_{n, j}\right\rangle+\frac{1}{r_{n}}\left\langle y-u_{n, j}, u_{n, j}-x_{n}\right\rangle \geq 0, \quad \forall y \in \mathscr{C} .
$$

This implies that

$$
\left\langle y-u_{n, j}, x_{n}-r_{n} A_{j} x_{n}-u_{n, j}\right\rangle \leq 0, \quad \forall y \in \mathscr{C},
$$

from which it follows that

$$
u_{n, j}=P_{\mathscr{C}}\left(x_{n}-r_{n} A_{j} x_{n}\right)
$$

This completes the proof.

Finally, we provide an example of GEP satisfying the conditions of Theorem 3.1, and give some numerical results to illustrate our algorithm.

Consider $X=\mathbb{R}$ and $\mathscr{C}=[\circ, 1]$. For any $i \in I$, we define the bifunctions $\Upsilon_{i}$ by

$$
\left\{\begin{array}{l}
\Upsilon_{i}: \mathscr{C} \times \mathscr{C} \longrightarrow \mathbb{R} \\
\Upsilon_{i}(x, y)=(y+i x)(y-x)
\end{array}\right.
$$


and $A_{i} x=i x$. It is easy to see that, for each $i \in I, \Upsilon_{i}$ satisfies the conditions $(\mathbf{C 1})-(\mathbf{C 4})$, also $A_{i}$, for each $i \in I$, is $\frac{1}{i+1}$-strongly monotone mapping. Indeed,

$$
\begin{aligned}
\left\langle A_{i} x-A_{i} y, x-y\right\rangle & =\langle i x-i y, x-y\rangle \\
& =i\langle x-y, x-y\rangle \\
& =i\|x-y\|^{2} \\
& \geq \frac{1}{i+1}\|x-y\|^{2} .
\end{aligned}
$$

Let $\left\{S_{n}(x):=\frac{x}{n+1}\right\}_{n \geq 1}$ be an infinite family of nonexpansive mappings on $\mathscr{C}$. For all $n \geq 1$, define the sequences $\left\{\alpha_{n}\right\},\left\{\beta_{n}\right\}$ and $\left\{\gamma_{n}\right\}$ in $(\circ, 1)$ by $\alpha_{n}=\frac{1}{n+1}, \beta_{n}=\frac{1}{n^{2}+1}$ and $\gamma_{n}=\frac{1}{n}$. Take $r_{n}=\frac{1}{n+1}$ and $f(x)=\frac{x}{3}$. Therefore, all conditions of Theorem 3.1 are satisfied. First, we find the sequence $\left\{u_{n, i}\right\}$ which satisfies the following Generalized Equilibrium Problem, for all $y \in \mathscr{C}$,

$$
\Upsilon_{i}\left(u_{n,}, y\right)+\left\langle A_{i} x_{n}, y-u_{n, i}\right\rangle+\frac{1}{r_{n}}\left\langle y-u_{n, i}, u_{n, i}-x_{n}\right\rangle \geq 0 .
$$

For all $n \geq 1$ and $i \in I$, we get

$$
\begin{aligned}
& \Upsilon_{i}\left(u_{n, i}, y\right)+\left\langle A_{i} x_{n}, y-u_{n, i}\right\rangle+\frac{1}{r_{n}}\left\langle y-u_{n, i}, u_{n, i}-x_{n}\right\rangle \geq 0 \\
& \Longleftrightarrow\left(y+i u_{n, i}\right)\left(y-u_{n, i}\right)+\left(i x_{n}\right)\left(y-u_{n, i}\right)+\frac{1}{r_{n}}\left(y-u_{n, i}\right)\left(u_{n, i}-x_{n}\right) \geq 0 \\
& \Longleftrightarrow r_{n} y^{2}-r_{n} u_{n, i} y+r_{n} i u_{n, i} y-r_{n} i u_{n, i}^{2}+r_{n} i x_{n} y-r_{n} i x_{n} u_{n, i}+y u_{n, i}-y x_{n}-u_{n, i}^{2}+u_{n, i} x_{n} \geq 0 \\
& \Longleftrightarrow r_{n} y^{2}+\left(\left(1+r_{n}(i-1) u_{n, i}\right)-\left(1-i r_{n}\right) x_{n}\right) y+\left(\left(1-i r_{n}\right) u_{n, i} x_{n}-\left(1+i r_{n}\right) u_{n, i}^{2}\right) \geq 0 .
\end{aligned}
$$

Put

$$
K_{i}(y)=r_{n} y^{2}+\left(\left(1+r_{n}(i-1) u_{n, i}\right)-\left(1-i r_{n}\right) x_{n}\right) y+\left(\left(1-i r_{n}\right) u_{n, i} x_{n}-\left(1+i r_{n}\right) u_{n, i}^{2}\right) .
$$

Since $K_{i}$ is a quadratic function relative to $y, K_{i}(y) \geq 0$ for all $y \in \mathscr{C}$, if and only if the coefficient of $y^{2}$ is positive and the discriminant $\triangle_{i} \leq 0$. But

$$
\begin{aligned}
\triangle_{i} & =\left(\left(1+r_{n}(i-1) u_{n, i}\right)-\left(1-i r_{n}\right) x_{n}\right)^{2}-4 r_{n}\left(\left(1-i r_{n}\right) u_{n, i} x_{n}-\left(1+i r_{n}\right) u_{n, i}^{2}\right) \\
& =\left(\left(1+r_{n}(i-1) u_{n, i}\right)-\left(1-i r_{n}\right) x_{n}\right)^{2} .
\end{aligned}
$$

So, $T_{r_{n}, i}\left(x_{n}\right)=\frac{1-i r_{n}}{1+r_{n}(i+1)} x_{n}$. It is easy to see that $\Upsilon_{i}$ for all $i \in I$, satisfies the conditions $(\mathbf{C 1})-(\mathbf{C 4})$. Next, we sketch the graph of $\Upsilon_{i}$ for $i=1,2, \cdots, 100$ in three-dimensional space (see Figure 1). We see that

$$
\left(\bigcap_{i=1}^{100} \operatorname{Fix}\left(S_{i}\right)\right) \cap\left(\bigcap_{j=1}^{100} \operatorname{GEP}\left(\Upsilon_{j}, A_{j}\right)\right)=\{\circ\} .
$$

In this case, $x_{n}$ converges to zero. Thus, $\left\{x_{n}\right\}_{n \geq 1}$ is convergent. 


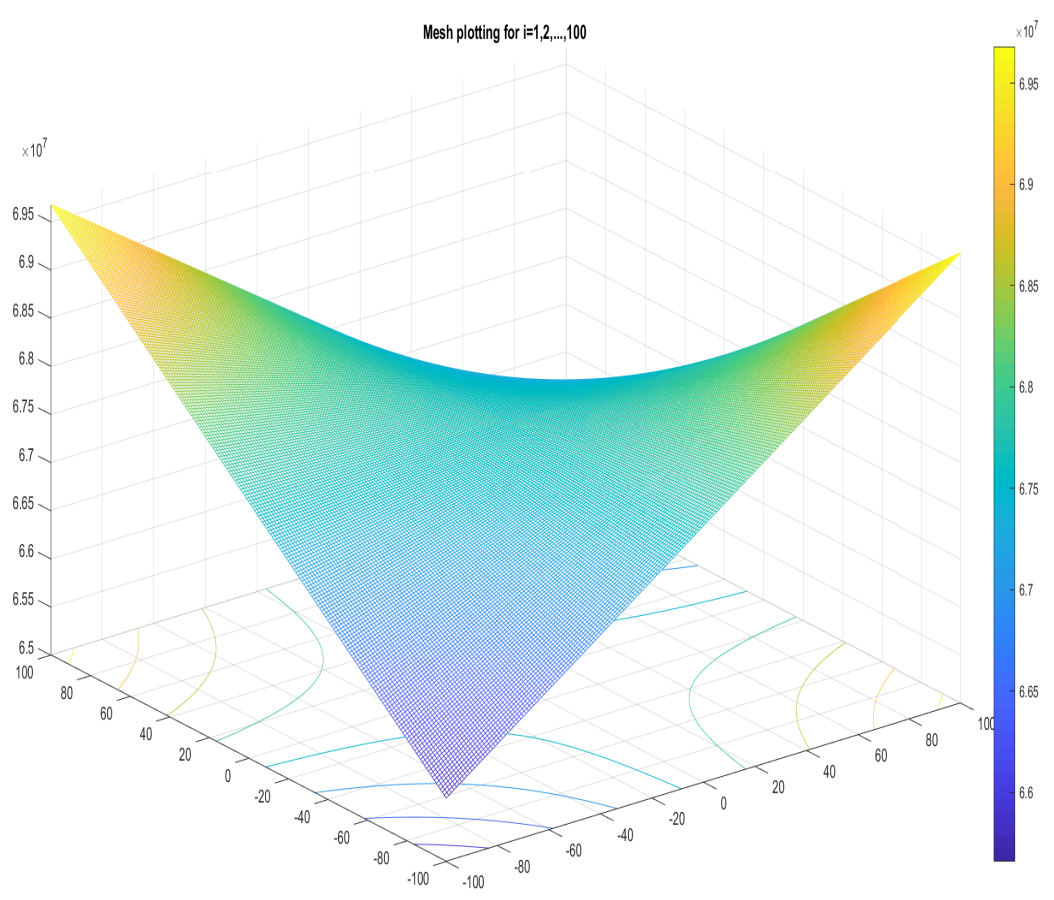

FIGURE 1. Sketched graph of $\Upsilon_{i}$ for $i=1,2, \cdots, 100$.

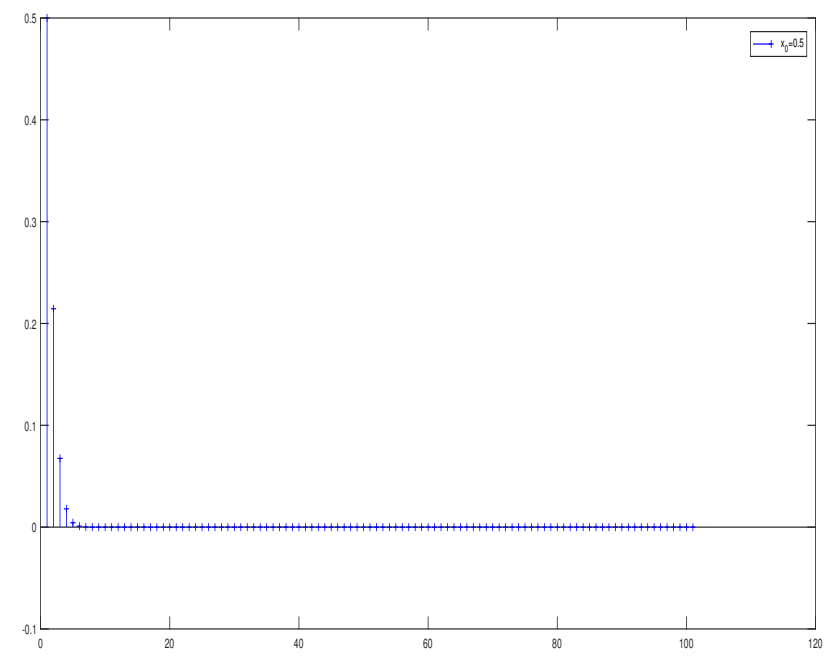

FIGURE 2. Plot of our algorithm.

\section{REFERENCES}

[1] K. Fan, A minimax inequality and applications, Academic Press, New York, 1972.

[2] E. Blum, W. Oettli, From optimization and variational inequlities to equilibrium problem, Math. student 63 (1994), 123145. 
[3] S.Y. Cho, X. Qin, On the strong convergence of an iterative process for asymptotically strict pseudocontractions and equilibrium problems, Appl. Math. Comput. 235 (2014), 430-438.

[4] S.Y. Cho, Generalized mixed equilibrium and fixed point problems in a Banach space, J. Nonlinear Sci. Appl. 9 (2016), 1083-1092.

[5] S.Y. Cho, B.A. Bin Dehaish, X. Qin, Weak convergence of a splitting algorithm in Hilbert spaces, J. Appl. Anal. Comput. 7 (2017), 427-438.

[6] B.A. Bin Dehaish, A regularization projection algorithm for various problems with nonlinear mappings in Hilbert spaces, J. Inequal. Appl. 2015 (2015), Article ID 51.

[7] A. Abkar, M. Tavakkoli, Convergence theorem for a system of equilibrium problems and a family of hybrid mappings, J. Nonlinear Convex Anal. 19 (2018), 853-865.

[8] X. Qin, Y.J. Cho, Projection splitting algorithms for nonself operators, J. Nonlinear Convex Anal. 18 (2017), 925-935.

[9] P. L. Combettes, S. A. Hirstoaga, Equilibrium programming in Hilbert spaces, J. Nonlinear Convex Anal. 6 (2005), 117136.

[10] Z. Lin, The study of traffic equilibrium problems with capacity constraints of arcs, Nonlinear Anal. 11 (2010), $2280-2284$.

[11] J. Li, Constrained ordered equilibrium problems and applications, J. Nonlinear Var. Anal. 1 (2017), 357-365.

[12] T. Ui, Bayesian Nash equilibrium and variational inequalities, J. Math. Economics 63 (2016), 139-146.

[13] L. Zhang, Y. Hao, Fixed point methods for solving solutions of a generalized equilibrium problem, J. Nonlinear Sci. Appl. 9 (2016), 149-159.

[14] P. Majee, C. Nahak, A modified iterative method for capturing a common solution of split generalized equilibrium problem and fixed point problem, Rev. R. Acad. Cienc. Exactas Fis. Nat. Ser. A Math. RACSAM 112 (2018), 1327-1348.

[15] X. Qin, S.Y. Cho, L. Wang, Strong convergence of an iterative algorithm involving nonlinear mappings of nonexpansive and accretive type, Optimization, 67 (2018), 1377-1388.

[16] X. Qin, S.Y. Cho, Convergence analysis of a monotone projection algorithm in reflexive Banach spaces, Acta Math. Sci. 37 (2017), 488-502.

[17] S. Takahashi, W. Takahashi, Strong convergence theorem for a generalized equilibrium problem and nonexpansive mapping in a Hilbert space, Nonlinear Anal. 69 (2008), 1025-1033.

[18] T.M. Tuyen, Parallel iterative methods for solving systems of generalized mixed equilibrium problems in reflexive Banach spaces, Optimization, 66 (2017), 623-639.

[19] K. Shimoji, W. Takahashi, Strong convergence to common fixed points of infinite nonexpansive mappings and applications, Taiwanese J. Math. 5 (2001), 387-404.

[20] S. S. Chang, H. W. J. Lee, C. K. Chan, A new method for solving equilibrium problem fixed point problem and variational inequality problem with application to optimization, Nonlinear Anal. 70 (2009), 3307-3319.

[21] X. Qin, Y.J. Cho, S.S. Chang, Iterative methods for generalized equilibrium problems and fixed point problems with applications Nonlinear Anal. 11 (2010), 2963-2972.

[22] Q.L. Dong, Y. Yao, An iterative method for finding common solutions of systems of equilibrium problems and fixed point problems in Hilbert spaces, An. Stiint. Univ. Ovidius Constanta, Ser. Mat. 19 (2011), 101-116.

[23] Z. Opial, Weak convergence of the sequence of successive approximations for nonexpansive mappings, Bull. Amer. Math. Soc. 73 (1967), 591-597.

[24] L. S. Liu, Ishikawa and Mann iteration process with errors for nonlinear strongly accretive mappings in Banach spaces, J. Math. Anal. Appl. 194 (1995), 114-125.

[25] T. Suzuki, Strong convergence of Krasnoselskii and Mann's type sequence for one-parameter nonexpansive semi-groups without Bochner integrals, J. Math. Anal. Appl. 305 (2005), 227-239. 